\title{
Quantum Molecular Dynamics Study on Energy Transfer to the Secondary Electron in Surface Collision Process of an Ion"
}

\author{
Masahiko SHIBAHARA $^{* *}$, Shin-ichi SATAKE ${ }^{* * *}$ and Jun TANIGUCHI ${ }^{* * *}$ \\ ** Osaka University, \\ 2-1 Yamadaoka, Suita, Osaka, Japan \\ E-mail: siba@mech.eng.osaka-u.ac.jp \\ *** Tokyo University of Science, \\ 2641 Yamazaki, Noda, Chiba, Japan
}

\begin{abstract}
In the present study the quantum molecular dynamics method was applied to an energy transfer problem to an electron during ionic surface collision process in order to elucidate how energy of ionic collision transfers to the emitted electrons. Effects of various physical parameters, such as the collision velocity and interaction strength between the observed electron and the classical particles on the energy transfer to the electron were investigated by the quantum molecular dynamics method when the potassium ion was collided with the surface so as to elucidate the energy path to the electron and the predominant factor of energy transfer to the electron. Effects of potential energy between the ion and the electron and that between the surface molecule and the electron on the electronic energy transfer were shown in the present paper. The energy transfer to the observed secondary electron through the potential energy term between the ion and the electron was much dependent on the ion collision energy although the energy increase to the observed secondary electron was not monotonous through the potential energy between the ion and surface molecules with the change of the ion collision energy.
\end{abstract}

Key words: Quantum Molecular Method, Electronic Dynamic Behavior, Focused Ion Beam, Energy Transfer, Time Dependent Schrödinger Equation

\section{Nomenclature}

$\mathrm{C}_{\mathrm{e}-\mathrm{xe}}$ : non-dimensional potential energy between a secondary electron and a surface molecule

$\mathrm{C}_{\mathrm{k}-\mathrm{e}} \quad$ : non-dimensional potential energy between an ion and a secondary electron

$\mathrm{C}_{4}, \mathrm{C}_{6}, \mathrm{C}_{9} \quad$ : potential parameters

$\Delta t \quad$ : time step

e : elementary electric charge

$\varepsilon \quad$ : a potential parameter

$\varepsilon_{0} \quad$ : vacuum dielectric constant

$\mathrm{H}$ : Hamiltonian of system

$\mathrm{H}_{\mathrm{a}}$ : Hamiltonian of classical particles

$\mathrm{H}_{\mathrm{e}} \quad$ : Hamiltonian of a secondary electron

$\hbar$ : Planck's constant/ $2 \pi$

$\mathrm{K} \quad$ : spatial differential term in a Schrödinger equation

$\mathrm{m}$ : mass of a secondary electron 


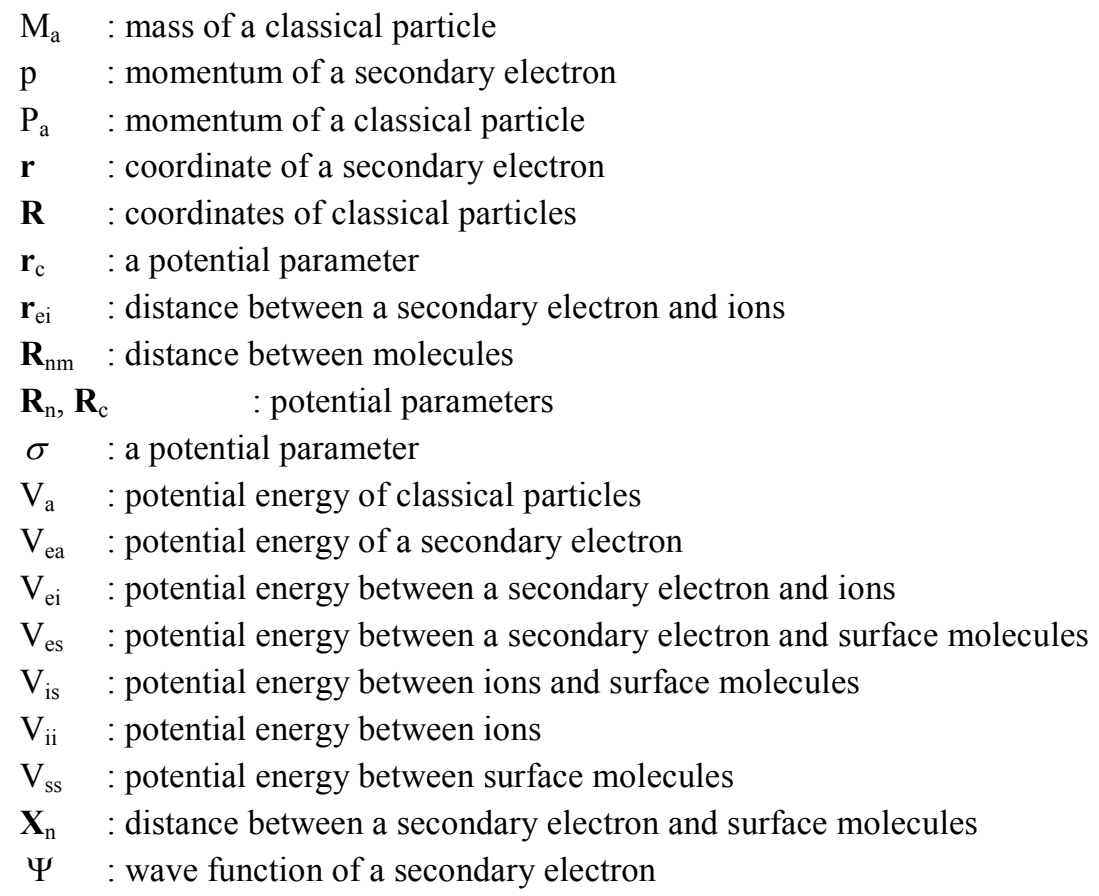

\section{Introduction}

It is well known that an emission of secondary electrons is observed in an ion collision process to a surface, such as the focused ion beam (FIB) process. Molecular dynamics simulations were sometimes conducted to predict the surface states during and after FIB process $^{(1)(2)}$. However, the physical effect of secondary emission on energy and mass transfer is seldom considered and there are few examples of analysis of the secondary electron emission. It is one of interesting problems as an extreme small scale energy transfer problem how energy is transferred to the electron emitted from the surface by ionic collisions. In the present study the quantum molecular dynamics method ${ }^{(3-6)}$ was applied to an energy transfer problem to an electron during ionic surface collision process in order to elucidate how energy of ionic collision transfers to the emitted electrons. As a quantum molecular dynamics method the Car-Parrinello method is well known and sometimes used for the system with periodic boundary conditions by using plane wave basis sets and density functional theory ${ }^{(7)}$. The quantum molecular dynamics method employed in the present study is a very primitive one in comparison with the Car-Parrinello method, however, the time dependent behavior of the electron can be solved by dealing with the electronic wave function as a wave packet. In the present paper, the energy transfer to the electron was discussed during the collision process of an ion with changing the interaction between the electron and ions and that between the electron and surface molecules by the quantum molecular dynamics method.

\section{Numerical Method}

In the present study, the Hamiltonian for the system $\mathrm{H}$ was assumed to be separable into the Hamiltonian of a secondary electron $\mathrm{H}_{\mathrm{e}}$ and that of classical particles $\mathrm{H}_{\mathrm{a}}$ as follows,

$$
H=H_{a}+H_{e} \text {. }
$$

The Hamiltonian for the observed secondary electron can be written as a summation of a kinetic energy term and a potential energy term,

$$
H_{e}=p^{2} /(2 m)+V_{e a}(\mathbf{r}, \mathbf{R}),
$$

where the first and the second terms on right hand side correspond to the kinetic energy term and the potential term of the electron, respectively. $\mathbf{r}$ and $\mathbf{R}$ represented the coordinates of the observed secondary electron and the classical particles, respectively. 
Wave function of the electron depending on time can be written as follows,

$$
|\Psi(t)\rangle=\exp \left(-i H_{e} t / \hbar\right)|\Psi(0)\rangle \text {. }
$$

In the present study, the wave function of the observed electron was expressed as a numerical function of complex numbers on $128 * 128$ grid points and equation (3) was solved by the split operator method ${ }^{(8)}$ so as to get time propagation of the wave function of the observed secondary electron as follows,

$$
\Psi(t+\Delta t)=\exp \left(\frac{-i \Delta t K}{2 \hbar}\right) \exp \left(-i V_{e a} \Delta t / \hbar\right) \exp \left(\frac{-i \Delta t K}{2 \hbar}\right) \Psi(t),
$$

where $\mathrm{K}$ corresponds to the spatial differential term. Fast Fourier Transform (FFT) method was employed for the spatial differential calculation in equation (4). The distance between grid points and the time interval for integration were $0.64 \AA$ and $0.01 \mathrm{fs}$, respectively.

The Hamiltonian for the classical particles $\mathrm{H}_{\mathrm{a}}$ can be written as follows,

$$
H_{a}=\sum_{a=1} P_{a}^{2} /\left(2 M_{a}\right)+V_{a}(\mathbf{R}),
$$

where "a" represents the classical particles such as ions and surface molecules in the system. In the present study, motions of the classical particles were calculated by solving the Newton's equations with the time interval of $0.2 \mathrm{fs}$. Hence, the equation (4) was time propagated for 20 steps when the Newton's equations for the classical particles were integrated for 1 step.

Figure 1 shows the calculation model employed in the present study and an example of initial probability density of an electron on grid points. We assumed that an ion (potassium) which had a certain velocity was collided with the other ion (potassium) with the observed secondary electron on the surface (xenon). The calculation model was different from a realistic FIB process so as to check the validity of the calculation methods in a simple system. The wave function of the observed electron was solved by equation (4) and the motions of ions and surface molecules were solved by the Newton's equations in order to investigate the behavior and energy transfer of the observed electron.

The potential functions between particles in the reference ${ }^{(3)}$ were employed in the present study and the potential parameters between the observed electron and an ion and those between the observed electron and surface molecules were treated as calculation parameters and changed from the standard value in the reference ${ }^{(3)}$. The potential functions employed are shown as follows,

$$
\begin{aligned}
& V_{e a}(\mathbf{r}, \mathbf{R})=V_{e i}+V_{e s}, \\
& V_{a}(\mathbf{R})=V_{i s}+V_{s s}+V_{i i}, \\
& V_{e i}(\mathbf{r})=-e^{2} /\left(4 \pi \varepsilon_{0} \mathbf{r}_{c}\right) \cdot C_{k-e} \quad\left|\mathbf{r}_{e i}\right|<\left|\mathbf{r}_{c}\right|=0.5 \dot{A} \\
& V_{e i}(\mathbf{r})=-e^{2} /\left(4 \pi \varepsilon_{0} \mathbf{r}_{e i}\right) \cdot C_{k-e}, \\
& V_{e s}=\sum_{n}\left(a / \mathbf{R}_{n i}^{4}\right)\left|b /\left(c+\mathbf{R}_{c}^{6}\right)-1\right| \cdot C_{e-x e}, \\
& \mathbf{R}_{n}=\max _{c}\left\{\mathbf{X}_{n}, \mathbf{R}_{c}\right\} \\
& \left.V_{s s}=(1 / 2) \sum_{n, m} 4 \varepsilon \mid-\left(\sigma / \mathbf{R}_{n m}\right)^{6}+\left(\sigma / \mathbf{R}_{n m}\right)^{12}\right], \\
& V_{i s}=\sum_{n}\left\lfloor C_{9} / \mathbf{R}_{i n}^{9}-C_{6} / \mathbf{R}_{i n}^{6}-C_{4} / \mathbf{R}_{i n}^{4}\right\rfloor \\
& V_{i i}(\mathbf{r})=e^{2} /\left(4 \pi \varepsilon_{0} \mathbf{r}\right),
\end{aligned}
$$

where $V_{\text {ea }}$ and $V_{a}$ were the potential function of the observed electron in equation (2) and that of the classical particles in equation (5), respectively. $\mathrm{V}_{\mathrm{ei}}, \mathrm{V}_{\mathrm{es}}, \mathrm{V}_{\mathrm{ss}}, \mathrm{V}_{\text {is }}$ and $\mathrm{V}_{\mathrm{ii}}$ were the potential function between the electron and the ions, that between the electron and surface molecules, that between surface molecules, that between the ions and the surface molecules and that between the ions, respectively. The effect of the electron coordinates in the potential energy was averaged in equation (7). Hence, $\mathrm{V}_{\mathrm{a}}$ was not a function of the 
observed electron coordinates $\mathbf{r}$. The standard parameters in equations (6)-(12) were the same as those in reference ${ }^{(3)}$. The magnitude of potential energy between the ion and the electron and that between the surface molecule and the electron were expressed as $\mathrm{C}_{\mathrm{k}-\mathrm{e}}$ and $\mathrm{C}_{\mathrm{e}-\mathrm{xe}}$ that were non dimensional value by dividing the standard value in the reference ${ }^{(3)}$.

\section{Results and Discussions}

Effects of various physical parameters, such as the collision velocity and interaction strength between the observed electron and the classical particles on energy transfer to the Table 1 Calculation parameters

\begin{tabular}{|c|c|c|}
\hline $\mathrm{C}_{\mathrm{k}-\mathrm{e}}$ & $\mathrm{C}_{\mathrm{e}-\mathrm{xe}}$ & Ion energy $\mathrm{E}_{\mathrm{i}}(\mathrm{eV})$ \\
\hline \hline 0.2 & 1.0 & \\
0.5 & 2.0 & 102 \\
0.75 & 3.0 & 914 \\
1.0 & 4.0 & 1829 \\
& 7.5 & \\
\hline
\end{tabular}

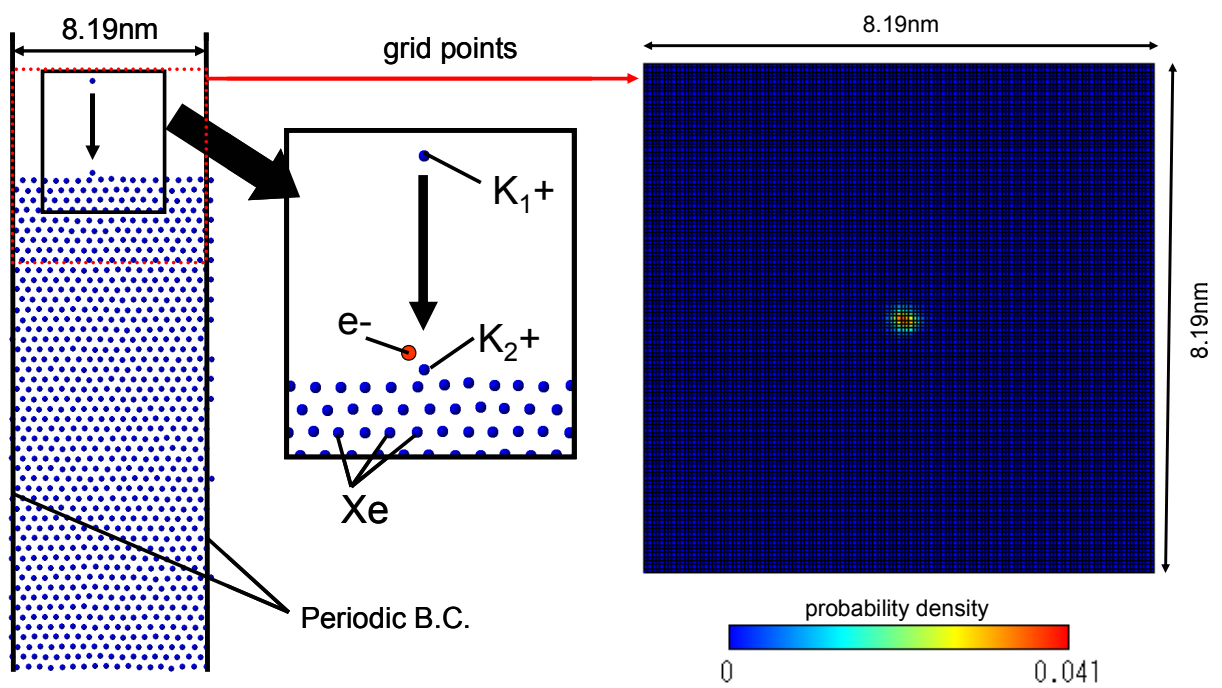

Fig.1 A calculation model for energy transfer to an electron by surface collision process of an ion and an example of initial probability density of an electron on grid points.

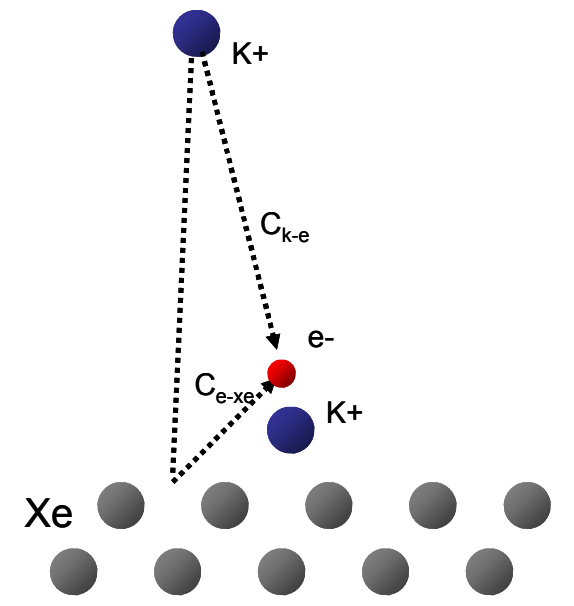

Fig.2 Energy transfer path to the observed secondary electron. 


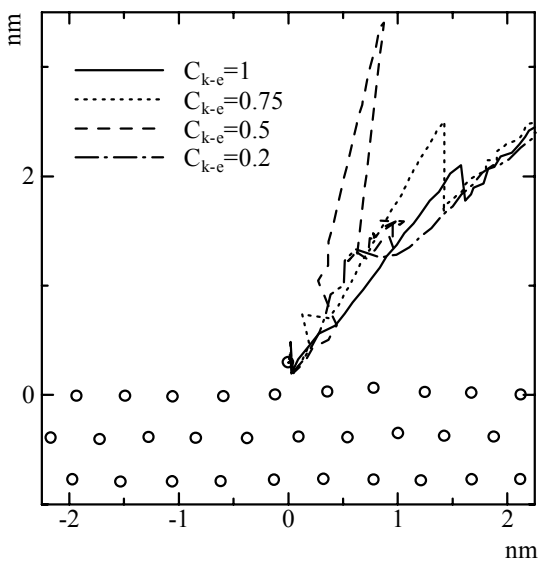

Fig. 3 Trajectories of the center of mass of the observed secondary electron during $1 \mathrm{ps} . \mathrm{C}_{\mathrm{e}-\mathrm{xe}}=1, \mathrm{E}_{\mathrm{i}}=102 \mathrm{eV}$.

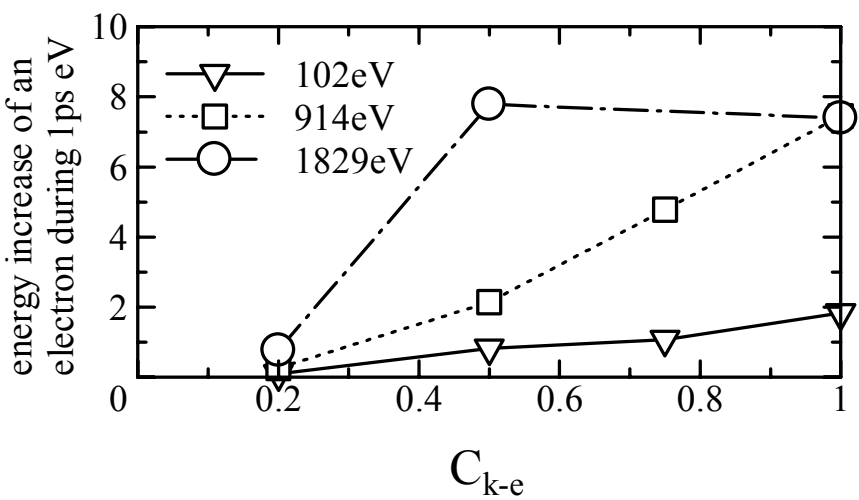

Fig.4 Effect of potential energy between ions and the observed secondary electron on the energy transfer to the observed secondary electron during $1.0 \mathrm{ps} . \mathrm{C}_{\mathrm{e}-\mathrm{xe}}=1$

observed secondary electron were investigated by the quantum molecular dynamics method when an ion was collided with the surface so as to elucidate the energy path to the electron and the predominant factor of energy transfer to the electron. Effects of potential energy between the ion and the electron and that between the surface molecule and the electron on the electronic energy transfer were shown in the present study. The ion collision velocity that can be converted to the ion translational energy is one of the important physical parameters. The calculation results were shown in the present report when the ion translational energy were 102, 914 and $1829 \mathrm{eV}$. The effects of $\mathrm{C}_{\mathrm{k}-\mathrm{e}}$ and $\mathrm{C}_{\mathrm{e}-\mathrm{xe}}$ on trajectories of the electronic center of mass and the energy transfer to the electron were calculated numerically. Figure 2 shows the considerable energy transfer path to the secondary electron through potential energy terms in equation (6) when the potential energy between the secondary electron and the ion on the surface was kept at constant. The magnitudes of $\mathrm{C}_{\mathrm{k}-\mathrm{e}}$ and $\mathrm{C}_{\mathrm{e}-\mathrm{xe}}$ were related to potential energy terms $\mathrm{V}_{\mathrm{ei}}$ and $\mathrm{V}_{\mathrm{es}}$ that were included in the time dependent Schrödinger equation (3), respectively. Hence, the secondary electron receives energy from the colliding ion through $\mathrm{V}_{\mathrm{ei}}$ or $\mathrm{V}_{\mathrm{es}}$.

\subsection{Effect of Potential Energy between Ions and the Secondary Electron on the Energy Transfer to the Electron}

Figure 3 shows the electronic trajectories during 1.0ps when the ion translational energy was $102 \mathrm{eV}$. Figure 4 shows effects of potential energy between ions and the secondary electron on the energy increase of the observed electron during $1 \mathrm{ps}$ when the magnitudes of $\mathrm{C}_{\mathrm{k}-\mathrm{e}}$ were changed from 0.2 to 1.0. When the ion collision energy were 102 and $914 \mathrm{eV}$, the energy of the observed electron increased monotonously with the increase of the 


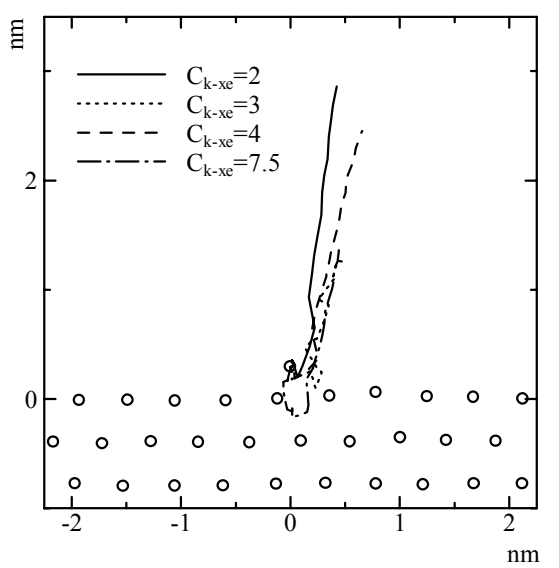

Fig. 5 Trajectories of the center of mass of the observed secondary electron during $1 \mathrm{ps} . \mathrm{C}_{\mathrm{k}-\mathrm{e}}=1, \mathrm{E}_{\mathrm{i}}=102 \mathrm{eV}$.

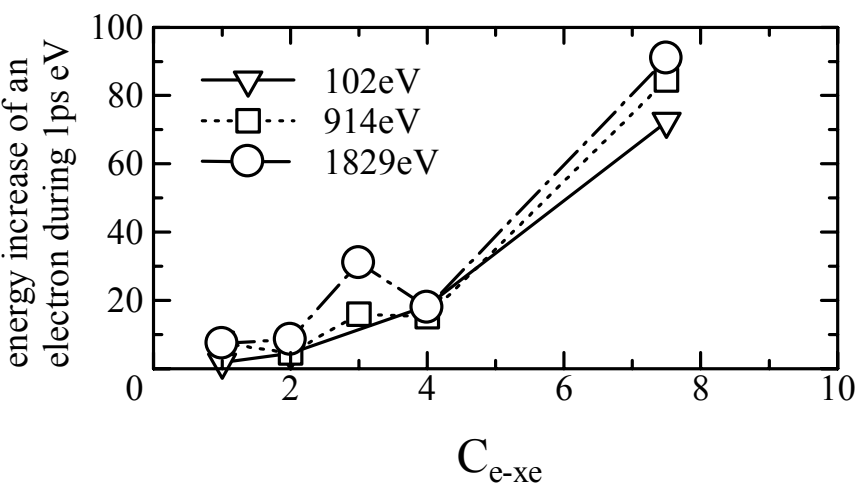

Fig.6 Effect of potential energy between surface molecules and the observed secondary electron on the energy transfer to the observed secondary electron during $1.0 \mathrm{ps} . \mathrm{C}_{\mathrm{k}-\mathrm{e}}=1$

magnitude of the potential energy between the ion and the electron, $\mathrm{C}_{\mathrm{k}-\mathrm{e}}$. When the ion translational energy was $1829 \mathrm{eV}$, the energy of the observed electron increased and became virtually constant. This is because there might be a limit of energy transfer to the observed electron during a constant time. In Figure 3 the trajectories of the observed electron were almost similar each other and the observed electron moved to upper right direction in all cases. This is because the electronic trajectories were greatly affected by the trajectories of the colliding ion as well as the surface ion. The trajectories of the ions were in the upper right direction under the present initial condition in all cases of $\mathrm{C}_{\mathrm{k}-\mathrm{e}}$. The wave function of the observed electron was expressed as a numerical function of complex numbers on grid points and the trajectories of the center of mass of the observed secondary electron were calculated by the time history of wave function that can be changeable much faster than motions of ions and surface molecules. The magnitude of potential energy between the ion and the electron, $C_{k-e}$, related to the magnitude of $V_{e i}$ in Equation (6). These results showed that the energy transfer to the observed electron from the colliding ion through the potential energy term between the ion and the electron, $V_{\text {ei }}$, was much dependent on the translation energy of the ion.

\subsection{Effect of Potential Energy between Surface Molecules and the Secondary Electron on the Energy Transfer to the Electron}

Figure 5 shows the trajectories of the center of mass of the observed secondary electron during $1.0 \mathrm{ps}$ when the ion translational energy was $102 \mathrm{eV}$. Figure 6 showed effects of potential energy between surface molecules and the secondary electron on the energy transfer to the observed secondary electron during $1 \mathrm{ps}$ when the magnitudes of $\mathrm{C}_{\mathrm{e}-\mathrm{xe}}$ were changed from 1 to 7.5 . In Figure 6 the energy of the observed electron increased with the 


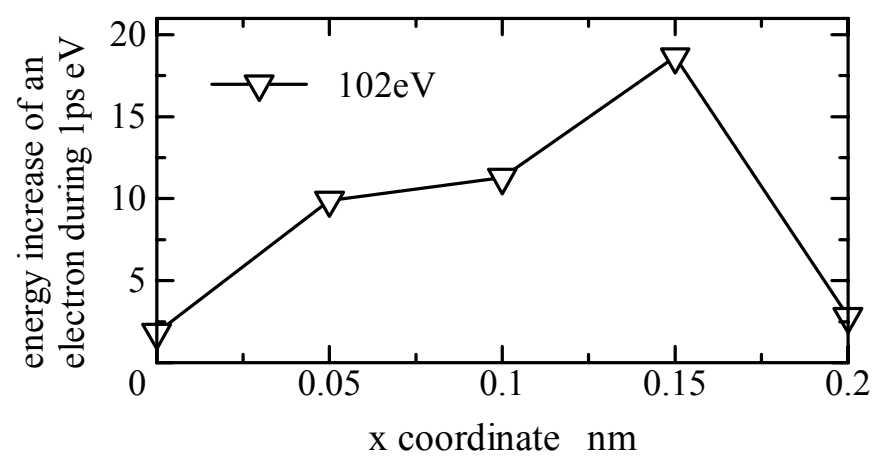

Fig.7 Effect of the colliding position on the energy transfer to the observed secondary electron during 1.0ps. $\mathrm{C}_{\mathrm{e}-\mathrm{xe}}=1, \mathrm{C}_{\mathrm{k}-\mathrm{e}}=1, \mathrm{E}_{\mathrm{i}}=102 \mathrm{eV}$.

increase of the magnitude of potential energy between surface molecules and the electron, $\mathrm{C}_{\mathrm{e}-\mathrm{xe}}$, when the ion translational energy were 102 and $914 \mathrm{eV}$. When the colliding energy of an ion was $1829 \mathrm{eV}$, the energy increase of the observed electron was not monotonous in comparison with the cases of 102 and $914 \mathrm{eV}$. The energy increase of the observed electron had fluctuation when the ion translational energy was relatively high. In Figure 6 the energy increase of the observed electron at low ion translational energy was relatively small in comparison with the case at high ion energy. However, the effect of the ion translational energy on the electronic energy increase was not monotonous when the magnitude of potential energy between the surface molecule and the electron $\mathrm{C}_{\mathrm{e}-\mathrm{xe}}$ was more than unity. When the potential energy between the surface molecule and the electron was changed as calculation parameters, the stable electronic distribution changed as initial conditions. Therefore the initial distribution of the electron probability was attracted to the surface molecules when $\mathrm{C}_{\mathrm{e}-\mathrm{xe}}$ was relatively large. On the other hand, the initial position of the colliding ion was fixed under all conditions. Hence, the effect of ion translational energy on the electronic energy increase was not monotonous with the change of the magnitude of the potential energy between surface molecules and the electron, $\mathrm{C}_{\mathrm{e}-\mathrm{xe}}$. In Figure 5 displacements of the center of mass of the observed electron became larger with the decrease of the magnitude of the potential energy between molecules and the electron, $\mathrm{C}_{\mathrm{e}-\mathrm{xe}}$. This is because the absolute value of the potential energy $\mathrm{V}_{\mathrm{es}}$ in Equation (6) became large with the increase of $\mathrm{C}_{\mathrm{e}-\mathrm{xe}}$. The trajectories of the ions were in the upper right direction under the present initial condition in all cases of $\mathrm{C}_{\mathrm{e}-\mathrm{xe}}$.

\subsection{Effect of colliding position on the energy transfer to the secondary electron}

Figure 7 shows the effect of the target position of the colliding ion on the energy transfer to the electron during $1 \mathrm{ps}$ when $\mathrm{C}_{\mathrm{e}-\mathrm{xe}}$ and $\mathrm{C}_{\mathrm{k}-\mathrm{e}}$ were unity. The origin of $\mathrm{x}$ coordinate was defined as the initial position of the ion on the surface and it can move freely before the collision of the ion. The target position of the colliding ion was changed from 0 to $0.2 \mathrm{~nm}$ in Figure 7. In Figure 7 the energy increase of the observed electron were much dependent on the surface colliding position. When the $\mathrm{x}$ coordinate was 0.15 $\mathrm{nm}$, the energy increase of the observed electron was 6 times as large as those at $\mathrm{x}=0 \mathrm{~nm}$ or $0.2 \mathrm{~nm}$. The electron distribution on the surface as well as the ion position on the surface can be changeable because the ion and surface molecules have thermal energy. Therefore, the colliding position of the ion was very important physical factor for the energy transfer of the secondary electron. When the $x$ coordinate was $0.15 \mathrm{~nm}$, we can suppose that the colliding position of the ion was just directly on the electronic distribution. The energy transfer to the observed electron was reduced when the target position was apart 
from $\mathrm{x}=0.15 \mathrm{~nm}$.

\section{Conclusions}

The quantum molecular dynamics method was applied to an energy transfer problem to a secondary electron during ionic surface collision process in order to elucidate how energy of the ionic collision transfers to the emitted electron from the surface. We assumed that an ion which had a certain translational velocity was collided with the other ion with the observed secondary electron on the surface. The wave function of the observed secondary electron was solved by the time dependent Schrödinger equation and the motions of ions and surface molecules were solved by the Newton's equations numerically. The calculation method employed in the present study can be applicable to an observation of a dynamic behavior of an electron that comes out from a surface during an ionic collision process such as FIB processes. The energy transfer paths to the electron in the time dependent Schrödinger equation was discussed during the collision process of an ion with changing the magnitude of potential energy between the electron and ions and that between the electron and surface molecules. The main findings are as follows:

(1)The energy transfer to the secondary electron from the colliding ion through the potential energy term between the ion and the electron was much dependent on the translation energy of the ion.

(2)The energy transfer to the secondary electron from the colliding ion through the potential energy term between the surface molecules and the electron was not monotonous with the change of the ion translational energy.

(3)The colliding position of the ion to the surface was very important physical factor for the energy transfer to the secondary electron.

\section{Acknowledgments}

This work was partially supported by the Ministry of Education, Science, Sports and Culture (MEXT), Japan, "Grant-in-Aid for Exploratory Research No. 18656066" and "Grant-in-Aid for Young Scientists (A) No. 19686017".

\section{References}

(1) S. Satake, N. Inoue, J. Taniguchi and M. Shibahara, Proceedings of Energy Nanotechnology International Conference 2006, No. ENIC2006-19061(2006), p.102.

(2) S. Satake, T. Kunugi, M. Shibahara, H. Kasahara and N. Inoue, Nuclear Instruments and Methods in Physics Research Section B: Beam Interactions with Materials and Atoms, Vol. B257 (2007), pp. 639-644.

(3) K. Haug and H. Metiu, Journal of Chemical Physics, Vol.99, No.9 (1993), pp.6253-6263.

(4) K. Haug and H. Metiu, Journal of Chemical Physics., Vol.97, No.7, (1992), pp.4781-4791.

(5) M. Shibahara and S. Kotake, International Journal of Heat and Mass Transfer, Vol.40, No.13, (1997), pp.3209-3222.

(6) M. Shibahara and S. Kotake, International Journal of Heat and Mass Transfer, Vol.41 Nos.6-7, (1998), pp.839-849.

(7) R. Car and M. Parrinello, Phys. Rev. Lett. 55, (1985), pp.2471 - 2474.

(8) M. D. Feit, J. A. Fleck, Jr. and Q. Steiger, Journal of Computational Physics, Vol. 47, (1982), pp.412-433. 